\title{
Constructing a New Frame of Spacelike Curves on Timelike Surfaces in Minkowski 3-Space
}

\author{
EMAD N. SHONODA \\ Faculty of Science, Port-Said University \\ Mathematics and Computer Science Department \\ EGYPT \\ Faculty of Science, Sohag University \\ Mathematics Department \\ EGYPT \\ Mt. San Jacinto College \\ Mathematics Department \\ California, USA \\ Faculty of Science, Islamic University of Madinah \\ Mathematics Department, 170 Al-Madinah \\ Saudi Arabia
}

\begin{abstract}
In this paper, we define a relative Minkowski normal plane $\zeta$ and relative tangent vector $T_{M}$. We construct a new relative $S$-frame (Shnoda-Saad frame) of regular spacelike curves on timelike surfaces. It depends only on the curve lies on the surface, Euclidean and Minkowski unit normal vectors. Also, we define $S$-curve according to thisframewithsomerelatedtheorems.
\end{abstract}

Key-Words: Spacelike curve, Timelike surface, Frenet and Darboux frames, Minkowski 3-space

Received: November 13, 2019. Revised: April 29, 2020. Accepted: May 14, 2020. Published: May 28, 2020.

\section{Introduction}

At the beginning of the 20th century, the Einstein's theory opened a door to new geometries such as Lorentzian Geometry, which is simultaneously the geometry of special relativity. After that, researchers discovered a bridge between modern differential geometry and the mathematical physics of general relativity. In particular, $E_{1}^{4}$ (Minkowski space-time) has a very strong background in physics. The Minkowski 3-space $E_{1}^{3} \subseteq E_{1}^{4}$ has many properties which is different from $E^{3}$. Some basic concepts such as vector, frame, and the motion of point have qualitative changes. Since the metric in $E_{1}^{3}$ is not positive definite metric, then the distance function $\langle$, can be positive, negative or zero, whereas the distance function in the Euclidean space can only be positive [1].

In Minkowski space $E_{1}^{3}$, every point in space and time worlds can be determined in terms of two special coordinates besides to the coordinate of time. When a point is moving along the time axis, it shouldn't move through space coordinates system. Moreover, it moves with the speed of light as a function of the time. Many authors tried to construct Darboux frame of a regular curve $\alpha(t)$ in $E_{1}^{3}$, which lies on a regular surface $\Phi$, by rotating Frenet frame $\{T, N, B\}$ around the tangent unit vector $T$ of $\alpha(t)[2,3,4]$. The curve $\alpha(t)$ is called Darboux curve if it moves on the surface $\Phi$ such that its curvature and normal curvature are identical. In other words, there is a transformation between Frenet frame $\{T, N, B\}$ and Darboux frame $\left\{T, N_{M}, G\right\}$, where $N_{M}$ and $G$ are the principal normal and binormal unit vectors of the surface $\Phi$ respectively. Furthermore, they are using other known frames, e.g., Sabban frame (Spherical Frenet) which is given according to Euclidean unit sphere $S^{2}$ [5]. Also, Bishop frame can be defined even if the curvature vanishes at some points on the curve $\alpha(t)[6,7,8]$.

Relative differential geometry can be described as the geometry of surfaces in the affine space with a distinguished relative normal vector field which gives us a general form of unit normal vector field from Euclidean differential geometry. In [9, 10, 11], the authors obtained a relative normal vector fields of a differentiable (smooth) surface which is defined as a function of two variables. The moving frame method provides a direct route to the classification of joint invariants and joint differential invariants [12]. There are some applications of moving frames in the construction of invariant numerical algorithms and the theory of geometric integration. Most modern Astronomy physics based on postulating a symmetry group and formulating field equations with a group-invariant variational principle. Furthermore, every invariant variational problem can be written in terms of the differential invariants of the symmetry group. The Euler-Lagrange equations inherit the symmetry group of the variational problem, which can be written in terms of the differential invariants. 
The general group-invariant formula construct the Euler-Lagrange equations from the invariant form of the variational problem $[13,14]$.

In this paper, we construct a new coordinate system by rotating the axes of space system about the time one. We should care that the axis of time rotates differently than the axes of space. Therefore, the rotation of the Frenet frame of $\alpha(t)$ which lies on a surface $\Phi$ in $E_{1}^{3}$ should be depend on the types of the curve, surface and axis of rotation. We call this frame "Shonoda-Saad frame" or simply S-frame.

This frame gives us a relative differential geometry between Euclidean and Minkowski invariants of a chosen curve on a regular timelike surface $\Phi$. We will investigate this special frame according to a regular spacelike curve $\alpha(t)$ on $\Phi$ in $E_{1}^{3}$.

\section{Differential Geometry Properties}

In this section, we list some notions, formulas, and conclusions for curves and surfaces in Minkowski 3 -space $E_{1}^{3}$ [15]. We consider the real vector space $E^{3}$ which defined the standard flat metric given by $\langle\rangle=,d x_{1}^{2}+d x_{2}^{2}-d x_{3}^{2}$, where $\left(x_{1}, x_{2}, x_{3}\right)$ is a rectangular coordinate system of $E_{1}^{3}$. Minkowski inner product of two vectors $\mathbf{u}, \mathbf{v} \in E_{1}^{3}$ is defined as $\langle\mathbf{u}, \mathbf{v}\rangle_{M}=u_{1} v_{1}+u_{2} v_{2}-u_{3} v_{3}$, where $\mathbf{u}=\left(u_{1}, u_{2}, u_{3}\right)$ and $\mathbf{v}=\left(v_{1}, v_{2}, v_{3}\right)$.

To distinguish the usual Euclidean inner product, we denote it by the notation $\langle,\rangle_{E}$. A vector $\mathbf{v} \in E_{1}^{3}$ is said to be spacelike, timelike or lightlike if $\langle\mathbf{v}, \mathbf{v}\rangle_{M}>0,\langle\mathbf{v}, \mathbf{v}\rangle_{M}<0$ or $\langle\mathbf{v}, \mathbf{v}\rangle_{M}=0$, respectively. The Minkowski norm of any vector $\mathbf{u} \in$ $E_{1}^{3}$ is defined as $\|\mathbf{u}\|_{M}=\sqrt{\left|\langle\mathbf{u}, \mathbf{u}\rangle_{M}\right|}$, which equals zero iff $\mathbf{u}$ is lightlike vector or $\mathbf{u}=0$. Furthermore, any two vectors $\mathbf{u}, \mathbf{v} \in E_{1}^{3}$ are orthogonal with the notation $\mathbf{u} \perp_{M} \mathbf{v}$ iff $\langle\mathbf{u}, \mathbf{v}\rangle_{M}=0$. The Lorentz vector product of $\mathbf{u}, \mathbf{v}$ is given by

$$
\mathbf{u} \times_{M} \mathbf{v}=\left|\begin{array}{ccc}
e_{1} & e_{2} & -e_{3} \\
u_{1} & u_{2} & u_{3} \\
v_{1} & v_{2} & v_{3}
\end{array}\right| .
$$

The light-cone is the set of all lightlike vectors of $E_{1}^{3}$. The timelike vector $\mathbf{v}=\left(v_{1}, v_{2}, v_{3}\right) \in E_{1}^{3}$ is called future (past) pointing iff $v_{3}>0\left(v_{3}<0\right)$, i.e. it lies in the future (past) section of the unit ball.

Definition 1 A surface $\Phi$ in the Minkowski 3-space $E_{1}^{3}$ is said to be spacelike, timelike surface if the induced metric on the surface is a positive definite Riemannian metric, Lorentz metric, respectively.
In other words, the normal vector on the spacelike (timelike) surface is a timelike (spacelike) vector [16].

Definition 2 Relative Minkowski normal plane $\zeta$ : Let $\Phi$ be a spacelike (timelike) surface with a unit timelike (spacelike) normal vector field $N_{M} \in E_{1}^{3}$ and Euclidean unit normal vector field $N_{E} \in E^{3}$. If $N_{M}$ and $N_{E}$ are non-congurent, then we have a plane $\zeta=$ $\operatorname{span}\left\{N_{E}, N_{M}\right\}$ which is called "relative Minkowski normal plane" of spacelike (timelike) surface $\Phi$.

Definition 3 Relative tangent vector $T_{M}$ : Consider a spacelike (timelike) surface $\Phi \in E_{1}^{3}$, then the tangent unit vector $T_{M}$ which generated from the intersection of the tangent plane and the relative Minkowski normal plane is called a relative tangent unit vector.

Definition 4 For a spacelike (timelike) surface $\Phi \in$ $E_{1}^{3}$, the frame $\left\{T_{M}, N_{M}, B_{M}\right\}$ is called ShonodaSaad frame or simply $\boldsymbol{S}$-frame where $B_{M}$ is a relative binormal vector field.

\subsection{Angle Measure in Lorentz-Minkowski Space $E_{1}^{3}$}

The angle measure between any two vectors $\mathbf{u}, \mathbf{v} \in E_{1}^{3}$ depends on the position of these vectors with respect to the light-cone $C=$ $\left\{\mathbf{u}=\left(x_{1}, x_{2}, x_{3}\right) \in E_{1}^{3}: x_{1}^{2}+x_{2}^{2}-x_{3}^{2}=0, \mathbf{u} \neq \mathbf{0}\right\}$. It can be determined only if both are timelike vectors lie in the same timelike cone or in the same spacelike component.

Definition 5 If $\mathbf{u} \in E_{1}^{3}$ is a timelike vector, then the timelike cone $C_{t}(\mathbf{u})$ is given as follows

$$
C_{t}(\mathbf{u})=\left\{\mathbf{v} \in \Gamma \subset E_{1}^{3}:\langle\mathbf{u}, \mathbf{v}\rangle_{M}<0\right\},
$$

where $\Gamma$ is the set of all timelike vectors in $E_{1}^{3}$. For more details see [17].

Corollary 6 If $\mathbf{u}, \mathbf{v} \in E_{1}^{3}$ are two timelike vectors lie in the same lightlike cone, then there exists a unique number $\varphi$ (the hyperbolic angle between $\mathbf{u}$ and $\mathbf{v}$ ) such that

$$
\langle\mathbf{u}, \mathbf{v}\rangle_{M}=-\|\mathbf{u}\|_{M}\|\mathbf{v}\|_{M} \cosh \varphi .
$$

Proof: The proof can be found in [17].

For any other two vectors, we have three possibilities depending on the type and position of the subspace which contains the two vectors $\operatorname{span}\{\mathbf{u}, \mathbf{v}\}$. Therefore, the induced metric should be Riemannian, 
Lorentzian or degenerate. If the plane is Riemannian, then the angle measure definition is determined as in Euclidean space $E^{3}$. If the plane is Lorentzian, then it is isometric to the Lorentz-Minkowski plane $E_{1}^{2}$ which is divided into four sections. Two of them are future and past timelike cone, the others are two spacelike components. Furthermore, we can't define the angle between two unit spacelike (timelike) vectors of $E_{1}^{2}$ that nor belong to the same component, neither the angle between a spacelike and timelike vectors. The definition of angle is discussed in details in $E_{1}^{2}$ by F. Catoni $[18,19]$. The third case appears when the plane containing both lightlike vectors. Therefore, the vectors shouldn't be timelike. So, we can't define the angle between two spacelike vectors.

Corollary 7 Let $\mathbf{u}, \mathbf{v} \in E_{1}^{2}$ be two spacelike vectors such that $\frac{\mathbf{u}}{\|\mathbf{u}\|_{M}}$ and $\frac{\mathbf{v}}{\|\mathbf{v}\|_{M}}$ lie in the same spacelike section. Then, there exists a unique number $\varphi$ (the hyperbolic angle between $\mathbf{u}$ and $\mathbf{v})$ such that

$$
\langle\mathbf{u}, \mathbf{v}\rangle_{M}=\|\mathbf{u}\|_{M}\|\mathbf{v}\|_{M} \cosh \varphi .
$$

\section{Frame Construction on Timelike Surface in $E_{1}^{3}$}

Consider a regular spacelike curve $\gamma(s)$ lies on a timelike surface $\Phi$ with a unit spacelike normal $N_{M} \in$ $E_{1}^{3}$. We have a moving orthonormal Frenet frame $\{T, N, B\}$ on $\gamma(s)$ with $T \perp_{M} N, N \perp_{M} B$ and $B \perp_{M} T$. From the definition of spacelike curve $\gamma$, the tangent vector $T$ is spacelike vector and one of $N$ or $B$ is timelike vector.

Additionally, we have Darboux frame $\left\{T, N_{M}, G\right\}$, where $G=-T \times_{M} N_{M}$ is a timelike vector the curve $\gamma(s)$. We don't know the behavior of the principal normal vector $N$. Therefore, we should discuss the cases of spacelike or timelike principal normal vector $N$.

Consider a frame $\left\{T_{P}^{M}, N_{M}, B_{M}\right\}$ at a point $P \in$ $\Phi$ or simply $\left\{T_{M}, N_{M}, B_{M}\right\}$, where $T_{M}$ is a relative tangent vector lies in the plane $\operatorname{span}\left\{N_{E}, N_{M}\right\}$. So, we have the following cases:

Case 8 When the principal normal vector $N$ is a timelike. Then, the Frenet equations are given in matrix form as follows

$$
\left(\begin{array}{l}
T^{\prime} \\
N^{\prime} \\
B^{\prime}
\end{array}\right)=\left(\begin{array}{lll}
0 & \kappa & 0 \\
\kappa & 0 & \tau \\
0 & \tau & 0
\end{array}\right)\left(\begin{array}{c}
T \\
N \\
B
\end{array}\right)
$$

If $N$ and $G$ lie in the same timelike cone. Then, we have the usual transformation between Frenet and Darboux frames;

$$
\left(\begin{array}{c}
T \\
N_{M} \\
G
\end{array}\right)=\left(\begin{array}{ccc}
1 & 0 & 0 \\
0 & -\sinh \theta & \cosh \theta \\
0 & \cosh \theta & -\sinh \theta
\end{array}\right)\left(\begin{array}{c}
T \\
N \\
B
\end{array}\right)
$$

where the hyperbolic angle $\theta=\angle_{H}(N, G)$.

Therefore, we get

$$
\left(\begin{array}{c}
T^{\prime} \\
N_{M}^{\prime} \\
G^{\prime}
\end{array}\right)=\left(\begin{array}{ccc}
0 & \kappa_{g} & \kappa_{n} \\
-\kappa_{g} & 0 & \tau_{g} \\
\kappa_{n} & \tau_{g} & 0
\end{array}\right)\left(\begin{array}{c}
T \\
N_{M} \\
G
\end{array}\right),
$$
$\frac{d \theta}{d s}$.

with $\kappa_{g}=\kappa \sinh \theta, \kappa_{n}=\kappa \cosh \theta$ and $\tau_{g}=\tau-$

In this case, we have two subcases according to the type of the relative tangent vector $T_{M}$.

On one hand, when $T_{M}$ is a spacelike vector. Also, $T$ and $T_{M}$ lie in the same spacelike component. Since $B_{M}$ should be a timelike vector. Then, we get

$$
\left(\begin{array}{c}
T \\
N_{M} \\
G
\end{array}\right)=\left(\begin{array}{ccc}
\cosh \alpha & 0 & \sinh \alpha \\
0 & 1 & 0 \\
\sinh \alpha & 0 & \cosh \alpha
\end{array}\right)\left(\begin{array}{c}
T_{M} \\
N_{M} \\
B_{M}
\end{array}\right),
$$

and

$\left(\begin{array}{c}T_{M} \\ N_{M} \\ B_{M}\end{array}\right)=\left(\begin{array}{ccc}\cosh \alpha & 0 & -\sinh \alpha \\ 0 & 1 & 0 \\ -\sinh \alpha & 0 & \cosh \alpha\end{array}\right)\left(\begin{array}{c}T \\ N_{M} \\ G\end{array}\right)$,

where $\alpha=L_{H}\left(T, T_{M}\right)$, so we have

$$
\begin{aligned}
& \left(\begin{array}{c}
T_{M} \\
N_{M} \\
B_{M}
\end{array}\right) \\
= & \left(\begin{array}{ccc}
\cosh \alpha & -\cosh \theta \sinh \alpha & \sinh \theta \sinh \alpha \\
0 & -\sinh \theta & \cosh \theta \\
-\sinh \alpha & \cosh \theta \cosh \alpha & -\sinh \theta \cosh \alpha
\end{array}\right) \\
& \left(\begin{array}{c}
T \\
N \\
B
\end{array}\right) .
\end{aligned}
$$

Differentiating (6) with respect to $s$ and using Frenet equations, we obtain

$$
\begin{aligned}
& \left(\begin{array}{c}
T_{M}^{\prime} \\
N_{M}^{\prime} \\
B_{M}^{\prime}
\end{array}\right) \\
= & \left(\begin{array}{ccc}
0 & \kappa_{g}^{1}-\tau_{g}^{2} & -\kappa_{n} \\
\tau_{g}^{2}-\kappa_{g}^{1} & 0 & \tau_{g}^{1}-\kappa_{g}^{2} \\
-\kappa_{n} & \tau_{g}^{1}-\kappa_{g}^{2} & 0
\end{array}\right) \\
& \left(\begin{array}{c}
T_{M} \\
N_{M} \\
B_{M}
\end{array}\right),
\end{aligned}
$$


where $\kappa_{g}^{1}=\kappa_{g} \cosh \alpha, \kappa_{g}^{2}=\kappa_{g} \sinh \alpha, \tau_{g}^{1}=$ $\tau_{g} \cosh \alpha, \tau_{g}^{2}=\tau_{g} \sinh \alpha$, and $-\kappa_{n}=\kappa_{n}-\frac{d \alpha}{d s}$.

On the other hand, if $T_{M}$ is a timelike vector. Then, $B_{M}$ should be spacelike vector. Therefore, we get

$$
\left(\begin{array}{c}
T \\
N_{M} \\
G
\end{array}\right)=\left(\begin{array}{ccc}
\sinh \beta & 0 & \cosh \beta \\
0 & 1 & 0 \\
\cosh \beta & 0 & \sinh \beta
\end{array}\right)\left(\begin{array}{c}
T_{M} \\
N_{M} \\
B_{M}
\end{array}\right),
$$

where $\beta=L_{H}\left(G, T_{M}\right)$. Then, we have

$$
\begin{aligned}
& \left(\begin{array}{l}
T \\
N \\
B
\end{array}\right) \\
= & \left(\begin{array}{ccc}
\sinh \beta & 0 & \cosh \beta \\
\cosh \theta \cosh \beta & \sinh \theta & \cosh \theta \sinh \beta \\
\sinh \theta \cosh \beta & \cosh \theta & \sinh \theta \sinh \beta
\end{array}\right) \\
& \left(\begin{array}{l}
T_{M} \\
N_{M} \\
B_{M}
\end{array}\right) .
\end{aligned}
$$

Using (7), by replacing the angle $\alpha$ by $\beta$, we obtain

$$
\begin{aligned}
& \left(\begin{array}{c}
T_{M}^{\prime} \\
N_{M}^{\prime} \\
B_{M}^{\prime}
\end{array}\right) \\
= & \left(\begin{array}{ccc}
0 & \kappa_{g}^{2}-\tau_{g}^{1} & -\kappa_{n} \\
\tau_{g}^{1}-\kappa_{g}^{2} & 0 & \tau_{g}^{2}-\kappa_{g}^{1} \\
-\kappa_{n} & \tau_{g}^{2}-\kappa_{g}^{1} & 0
\end{array}\right)\left(\begin{array}{c}
T_{M} \\
N_{M} \\
B_{M}
\end{array}\right),
\end{aligned}
$$

where $\kappa_{g}^{1}=\kappa_{g} \cosh \beta, \kappa_{g}^{2}=\kappa_{g} \sinh \beta, \tau_{g}^{1}=$ $\tau_{g} \cosh \beta, \tau_{g}^{2}=\tau_{g} \sinh \beta$, and $-\kappa_{n}=\kappa_{n}-\frac{d \beta}{d s}$.

Definition 9 Let $\Phi$ be a timelike surface in $E_{1}^{3}$ and $\gamma(s)$ be a regular spacelike curve lies on $\Phi$ with timelike principal normal vector $N$. The curve $\gamma(s)$ is called a spaelike S-curve of first or second type, if $\frac{\tau_{g}}{\kappa_{g}}=\tanh \psi$ or $\frac{\kappa_{g}}{\tau_{g}}=\tanh \psi, \psi=\alpha$ or $\beta, \psi \neq 0$, respectively.

Theorem 10 Let $\Phi$ be a timelike surface in $E_{1}^{3}$ and $\gamma(s)$ a regular spacelike $S$-curve of first or second kind with a timelike principal normal vector $N$. Then, the $S$ - frame $\left\{T_{M}, N_{M}, B_{M}\right\}$ of $\gamma(s)$ is Bishop frame of first or second type, respectively.

Proof: Without loss of generality, consider a case of $T_{M}$ as a spacelike vector. If $\frac{\tau_{g}}{\kappa_{g}}=\tanh \alpha$, then we have $\tau_{g}^{1}=\kappa_{g}^{2}$. Therefore, we can rewrite (7) as follows

$$
\left(\begin{array}{c}
T_{M}^{\prime} \\
N_{M}^{\prime} \\
B_{M}^{\prime}
\end{array}\right)=\left(\begin{array}{ccc}
0 & \frac{\kappa_{g}}{\cosh \alpha} & -\kappa_{n} \\
-\frac{\kappa_{g}}{\cosh \alpha} & 0 & 0 \\
-\kappa_{n} & 0 & 0
\end{array}\right)\left(\begin{array}{c}
T_{M} \\
N_{M} \\
B_{M}
\end{array}\right),
$$

which is a new Bishop frame of the first type relative to the surface $\Phi$. Furthermore, if $\frac{\kappa_{g}}{\tau_{g}}=\tanh \alpha$ then we have $\kappa_{g}^{1}=\tau_{g}^{2}$. Therefore, we can write (7) as follows

$$
\left(\begin{array}{c}
T_{M}^{\prime} \\
N_{M}^{\prime} \\
B_{M}^{\prime}
\end{array}\right)=\left(\begin{array}{ccc}
0 & 0 & -\kappa_{n} \\
0 & 0 & \frac{\tau_{g}}{\cosh \alpha} \\
-\kappa_{n} & \frac{\tau_{g}}{\cosh \alpha} & 0
\end{array}\right)\left(\begin{array}{c}
T_{M} \\
N_{M} \\
B_{M}
\end{array}\right)
$$

which is a new Bishop frame of the second type relative to the surface $\Phi$. Similarly, if $T_{M}$ is a timelike vector, we can get the same results.

Case 11 When the principal normal vector $N$ is a spacelike vector. So, the vector $B$ should be timelike vector. Then, the Frenet equations is given as follows

$$
\left(\begin{array}{l}
T^{\prime} \\
N^{\prime} \\
B^{\prime}
\end{array}\right)=\left(\begin{array}{ccc}
0 & \kappa & 0 \\
-\kappa & 0 & \tau \\
0 & \tau & 0
\end{array}\right)\left(\begin{array}{l}
T \\
N \\
B
\end{array}\right)
$$

If $N$ and $N_{M}$ are lie in the same spacelike sector with the hyperbolic angle $\theta=L_{H}\left(N, N_{M}\right)$. Then, we have the usual transformation between Frenet and Darboux frames;

$$
\left(\begin{array}{c}
T \\
N_{M} \\
G
\end{array}\right)=\left(\begin{array}{ccc}
1 & 0 & 0 \\
0 & \cosh \theta & -\sinh \theta \\
0 & -\sinh \theta & \cosh \theta
\end{array}\right)\left(\begin{array}{c}
T \\
N \\
B
\end{array}\right),
$$

and then, we get

$$
\left(\begin{array}{c}
T^{\prime} \\
N_{M}^{\prime} \\
G^{\prime}
\end{array}\right)=\left(\begin{array}{ccc}
0 & \kappa_{n} & \kappa_{g} \\
-\kappa_{n} & 0 & \tau_{g} \\
\kappa_{g} & \tau_{g} & 0
\end{array}\right)\left(\begin{array}{c}
T \\
N_{M} \\
G
\end{array}\right) .
$$

In this case, we have two subcases according to the type of the relative tangent vector $T_{M}$.

Firstly, when $T_{M}$ is a spacelike vector and also $T$ and $T_{M}$ lie in the same spacelike component. Then, $B_{M}$ should be a timelike vector. Therefore, we get

$$
\left(\begin{array}{c}
T \\
N_{M} \\
G
\end{array}\right)=\left(\begin{array}{ccc}
\cosh \alpha & 0 & \sinh \alpha \\
0 & 1 & 0 \\
\sinh \alpha & 0 & \cosh \alpha
\end{array}\right)\left(\begin{array}{c}
T_{M} \\
N_{M} \\
B_{M}
\end{array}\right),
$$

and $\left(\begin{array}{c}T_{M} \\ N_{M} \\ B_{M}\end{array}\right)=\left(\begin{array}{ccc}\cosh \alpha & 0 & -\sinh \alpha \\ 0 & 1 & 0 \\ -\sinh -\alpha & 0 & \cosh -\alpha\end{array}\right)\left(\begin{array}{c}T \\ N_{M} \\ G\end{array}\right)$, 
where $\alpha=L_{H}\left(T, T_{M}\right)$. Then, we obtain

$$
\begin{aligned}
& \left(\begin{array}{l}
T_{M} \\
N_{M} \\
B_{M}
\end{array}\right) \\
= & \left(\begin{array}{ccc}
\cosh \alpha & \sinh \theta \sinh \alpha & -\cosh \theta \sinh \alpha \\
0 & \cosh \theta & -\sinh \theta \\
-\sinh \alpha & -\sinh \theta \cosh \alpha & \cosh \theta \cosh \alpha
\end{array}\right) \\
& \left(\begin{array}{c}
T \\
N \\
B
\end{array}\right) .
\end{aligned}
$$

Using Frenet and Darboux formulas lead to

$$
\begin{aligned}
& \left(\begin{array}{c}
T_{M}^{\prime} \\
N_{M}^{\prime} \\
B_{M}^{\prime}
\end{array}\right) \\
= & \left(\begin{array}{ccc}
0 & \kappa_{n}^{1}-\tau_{g}^{2} & -\kappa_{g} \\
\tau_{g}^{2}-\kappa_{n}^{1} & 0 & \tau_{g}^{1}-\kappa_{n}^{2} \\
-\kappa_{g} & \tau_{g}^{1}-\kappa_{n}^{2} & 0
\end{array}\right)\left(\begin{array}{l}
T_{M} \\
N_{M}(1 \\
B_{M}
\end{array}\right),
\end{aligned}
$$

where $\kappa_{n}^{1}=\kappa_{n} \cosh \alpha, \kappa_{n}^{2}=\kappa_{n} \sinh \alpha$, and $-\kappa_{g}=\kappa_{g}-\frac{d \alpha}{d s}$.

Secondly, if $T_{M}$ is a timelike vector. Then, $B_{M}$ should be a spacelike vector. Therefore, we get

$$
\left(\begin{array}{c}
T \\
N_{M} \\
G
\end{array}\right)=\left(\begin{array}{ccc}
\sinh \beta & 0 & \cosh \beta \\
0 & 1 & 0 \\
\cosh \beta & 0 & \sinh \beta
\end{array}\right)\left(\begin{array}{c}
T_{M} \\
N_{M} \\
B_{M}
\end{array}\right),
$$

where $\beta=L_{H}\left(G, T_{M}\right)$ and then we have

$$
\begin{aligned}
& \left(\begin{array}{l}
T \\
N \\
B
\end{array}\right) \\
= & \left(\begin{array}{ccc}
-\sinh \beta & -\cosh \beta \sinh \theta & \cosh \beta \cosh \theta \\
0 & \cosh \theta & -\sinh \theta \\
\cosh \beta & \sinh \beta \sinh \theta & -\sinh \beta \cosh \theta
\end{array}\right) \\
& \left(\begin{array}{c}
T_{M} \\
N_{M} \\
B_{M}
\end{array}\right) .
\end{aligned}
$$

Similarly, as (10) by replacing the angle $\alpha$ by $\beta$, we get

$$
\begin{aligned}
& \left(\begin{array}{c}
T_{M}^{\prime} \\
N_{M}^{\prime} \\
B_{M}^{\prime}
\end{array}\right) \\
= & \left(\begin{array}{ccc}
0 & \kappa_{n}^{2}-\tau_{g}^{1} & -\kappa_{g} \\
\tau_{g}^{1}-\kappa_{n}^{2} & 0 & \tau_{g}^{2}-\kappa_{n}^{1} \\
-\kappa_{g} & \tau_{g}^{2}-\kappa_{n}^{1} & 0
\end{array}\right)\left(\begin{array}{c}
T_{M} \\
N_{M} \\
B_{M}
\end{array}\right),
\end{aligned}
$$

where $\kappa_{n}^{1}=\kappa_{n} \cosh \beta, \kappa_{n}^{2}=\kappa_{n} \sinh \beta$, and $-\kappa_{g}=$ $\kappa_{g}-\frac{d \beta}{d s}$.

Definition 12 Let $\Phi$ be a timelike surface in $E_{1}^{3}$ and $\gamma(s)$ be a regular spacelike curve lies on $\Phi$ with a spacelike principal normal vector $N$. The curve $\gamma(s)$ is called a spaelike $S$-curve of third or fourth type if $\frac{\tau_{g}}{\kappa_{n}}=\tanh \psi$ or $\frac{\kappa_{n}}{\tau_{g}}=\tanh \psi ; \psi=\alpha$ or $\beta, \psi \neq 0$, respectively.

Theorem 13 Let $\Phi$ be a timelike surface in $E_{1}^{3}$ and $\gamma(s)$ be a regular spacelike $S$-curve of third or fourth type with spacelike principal normal vector $N$. Then, the $S$-frame $\left\{T_{M}, N_{M}, B_{M}\right\}$ of $\gamma(s)$ is Bishop frame of the first or second type, respectively.

Proof: Without loss of generality, consider a case of $T_{M}$ as a spacelike vector. If $\frac{\tau_{g}}{\kappa_{n}}=\tanh \alpha$, then we have $\kappa_{n}^{2}=\tau_{g}^{1}$. Therefore, we can rewrite (11) as follows

$$
\left(\begin{array}{c}
T_{M}^{\prime} \\
N_{M}^{\prime} \\
B_{M}^{\prime}
\end{array}\right)=\left(\begin{array}{ccc}
0 & \frac{\kappa_{n}}{\cosh \alpha} & -\kappa_{g} \\
-\frac{\kappa_{n}}{\cosh \alpha} & 0 & 0 \\
-\kappa_{g} & 0 & 0
\end{array}\right)\left(\begin{array}{c}
T_{M} \\
N_{M} \\
B_{M}
\end{array}\right),
$$

which is a new Bishop frame of the first type relative to the surface $\Phi$. Furthermore, if $\frac{\kappa_{n}}{\tau_{g}}=\tanh \alpha$, then we have $\tau_{g}^{2}=\kappa_{n}^{1}$. So, we can rewrite (11) as follows

$$
\left(\begin{array}{c}
T_{M}^{\prime} \\
N_{M}^{\prime} \\
B_{M}^{\prime}
\end{array}\right)=\left(\begin{array}{ccc}
0 & 0 & -\kappa_{g} \\
0 & 0 & \frac{\tau_{g}}{\cosh \alpha} \\
-\kappa_{g} & \frac{\tau_{g}}{\cosh \alpha} & 0
\end{array}\right)\left(\begin{array}{c}
T_{M} \\
N_{M} \\
B_{M}
\end{array}\right)
$$

which is a new Bishop frame of the second type relative to the surface $\Phi$. Similarly, if $T_{M}$ is a timelike vector, we obtain the same results.

Corollary 14 Let $\Phi$ be a timelike surface in $E_{1}^{3}$. Then, the $S$-frame $\left\{T_{M}, N_{M}, B_{M}\right\}$ and Darboux frame are identical iff $\zeta \cap T_{\Phi}^{P}=T$, where $P$ is a point lies on any regular curve $\gamma(s) \subset \Phi$.

Proof: Since $\zeta \cap T_{\Phi}^{P}=T$ iff the angle $\alpha=0$, then we have $\beta=0$ and hence $T_{M} \equiv T$ for all pervious cases.

\section{Conclusion}

In the three-dimensional Minkowski space $E_{1}^{3}$, a suitable differential equations of an $\mathrm{S}$-frame of a regular spacelike curve $\gamma(s)$ lies on a timelike surface $\Phi$ relative to $E_{1}^{3}$ according to a relative Euclidean and 
Minkowski normal vectors of $\Phi$ have been obtained. Also, some new concepts associated to $\mathrm{S}$-frame in addition to new invariants have been defined. Moreover, we have generated a so-called S-curve for some special values of hyperbolic angles. Finally, we have found that Bishop frame and S-frame are identical for S-curve of different types.

In the future studies, we will try to apply this frame on some special curves of a regular spacelike curve lying on spacelike and timelike surfaces in $E_{1}^{3}$. Hopefully, S-frame will be helpful to reconstruct and modify most of Minkowski geometry research ideas.

Acknowledgements: We would like to thank Prof. Gunter Weiss, University of Technology, Vienna, Austria for his guidance in this study. Also, we are very grateful to referees for the useful suggestions and remarks for the revised version.

\section{References}

[1] S. Izumiya, K. Saji and N. Takeuchi. Circular surfaces, Advances in Geometry 7(2), 2007, pp. 295-313.

[2] A. A. Ergin, On the generalized Darboux curves, Commun. Fac. Sci. Univ. Ank. Series $A_{1}$ 41, 1992, pp. 73-77.

[3] A. A. Ergin, Timelike Darboux curves on a timelike surface $\mathrm{M} \subset \mathrm{M}_{1}^{3}$, Hadronic Journal, 2001, pp. 701-712.

[4] M. Özdemir and A. A. Ergin, Spacelike Darboux curves in Minkowski 3-space, Differential Geometry - Dynamical Systems 9, 2007, pp. 131137.

[5] S. Şenyurt and A. Çalışkan, Spinor Formulation of Sabban Frame of Curve on $\mathrm{S}^{2}$, Pure Mathematical Sciences 4 (1), 2015, pp. 37-42.

[6] S. Yılmaz, Bishop spherical images of a spacelike curve in Minkowski 3-space, Int. Jour. Phys. Scien. 5(6), 2010, pp. 898-905.

[7] M. K. Karacan and B. Bükcü, Bishop frame of the timelike curve in Minkowski 3-space, Fen Derg. 3(1), 2008, pp. 80-90.

[8] O. J. Garay and A. Pámpano, Binormal Evolution of Curves with Prescribed Velocity, WSEAS Transactions on Fluid Mechanics 11, 2016, pp. 112-120.

[9] F. Manhart, Uneigentliche Relativsphären, die Regelflächen oder Rückungsflächen sind. Proceedings of the Congress of Geometry, Thessaloniki, 1987, pp. 106-113.
[10] F. Manhart, Eigentliche Relativsphären, die Regelflächen oder Rückungsflächen sind. Anz. Österr. Akad. Wiss. Math.-Naturwiss. Kl., 1988, pp. 37-40.

[11] F. Manhart, Relativgeometrische Kennzeichnungen euklidischer Hypersphären. Geom. Dedicata, 29, 1989, pp. 193-207.

[12] E. Calabi, P. J. Olver, C. Shakiban, A. Tannenbaum, and S. Haker, Differential and numerically invariant signature curves applied to object recognition, Int. J. Computer Vision 26, 1998, pp. 107-135.

[13] I.A. Kogan and P.J. Olver, Invariant EulerLagrange equations and the invariant variational bicomplex, Acta Appl. Math. 76, 2003, pp. 137193

[14] V. Itskov, Orbit reduction of exterior differential systems and group-invariant variational problems, Contemp. Math. 285, 2001, pp. 171-181.

[15] M. P. Do Carmo, Differential Geometry of Curves and Surface. Prentice-Hall, Englewood Cliffs, NJ, 1976.

[16] J. Suk Ro and D. Won Yoon, Tubes of Weingarten types in a Euclidean 3-space, J. Chungcheong Mathematical Society 22(3), 2009, pp. 359-366.

[17] R. López, Differential geometry of curves and surfacces in Lorentz-Minkowski space. Int. Elec. Journ. Geom. 3(2), 2010, pp. 67-101.

[18] F. Catoni, et al., The Mathematics of Minkowski Space-Time, Birkhäuser Verlag AG., 2008, ISBN 978-3-7643-8613-9.

[19] F. Catoni, et al., Geometry of Minkowski SpaceTime, Springer Science \& Business Media, 2011, ISBN 978-3-642-17977-8. 